\title{
Multiple lipomas linked to an RB1 gene mutation in a large pedigree with low penetrance retinoblastoma
}

\author{
Maurizio Genuardi ${ }^{1}$, Martina Klutz ${ }^{2}$, Koen Devriendt ${ }^{3}$, Daniela Caruso ${ }^{4}$, Mario Stirpe ${ }^{5}$ and \\ Dietmar R Lohmann*,2
}

${ }^{1}$ Medical Genetics, "A. Gemelli" School of Medicine, Catholic University, Rome, Italy; ${ }^{2}$ Institut für Humangenetik, Universitätsklinikum Essen, Essen, Germany; ${ }^{3}$ Center for Human Genetics, University of Leuven, Leuven, Belgium; ${ }^{4}$ Clinica Pediatrica, Università degli Studi di Catania, Italy; ${ }^{5}$ Fondazione G.B. Bietti per l'Oftalmologia, Rome, Italy

Hereditary predisposition to lipomas is observed in familial multiple lipomatosis (OMIM 151900) and benign cervical lipomatosis (OMIM 151800) and can also be associated with mutations in the MEN1 and PTEN genes (OMIM 131100 and 153480, respectively). In addition, a recent report indicates that a few patients with hereditary retinoblastoma also have lipomas. Here we report on an extended family segregating a splice site mutation in the RB1 gene. Almost all adult carriers of this mutation had multiple lipomas while penetrance for retinoblastoma was incomplete. In an unrelated pedigree, which was reported previously, the identical mutation was only associated with low-penetrance retinoblastoma but not lipomas. Our data indicate that lipoma predisposition in hereditary retinoblastoma is not associated with specific RB1 gene mutations but is influenced by modifying factors linked to this gene. European Journal of Human Genetics (2001) 9, 690-694.

Keywords: retinoblastoma; multiple lipoma; hereditary tumour predisposition; tumour suppressor genes; lipomatosis

\section{Introduction}

Hereditary retinoblastoma (OMIM 180200) is caused by mutations in the RB1 gene. Most germline mutations identified so far result in premature termination codons or loss of significant portions of the coding sequence. ${ }^{1}$ Rare mutant RB1 alleles have retained some of the functions of the normal allele and are associated with unilateral retinoblastoma and incomplete penetrance in families (low-penetrance retinoblastoma). Patients with bilateral retinoblastoma and, to a lesser extent, patients with unilateral retinoblastoma have an increased risk of death from second primary neoplasms, particularly sarcomas of bone and connective tissue, skin melanoma and brain tumours. ${ }^{2}$ These neoplasms occur only in some patients and thus it was hypothesised that

*Correspondence: Dietmar R Lohmann, Institut für Humangenetik, Universitätsklinikum Essen, Hufelandstrasse 55, D-45122 Essen, Germany. Tel: +49 201723 4562; Fax: +49 201723 5900;

E-mail: dr.lohmann@uni-essen.de

Received 12 February 2001; revised 30 May 2001; accepted 6 June 2001 second tumours are caused by specific germline mutations. ${ }^{2,3}$ So far, however, second tumours were not found to be associated with specific oncogenic RB1 gene mutations. ${ }^{1}$

Recently, Li and co-workers found that among 556 patients with hereditary retinoblastoma, 20 (3.6\%) reported diagnoses of subcutaneous lipoma, mostly as solitary tumours in the region of neck or back. ${ }^{4}$ Solitary lipomas are relatively common, essentially benign neoplasms of adipose tissue. Familial occurrence of multiple lipomas is rare and can be observed as an autosomal dominant trait with almost complete penetrance in two clinically distinct varieties, namely familial multiple lipomatosis (OMIM 151900) and benign cervical lipomatosis (OMIM 151800). These conditions can be discerned as the tumours show distinct local distribution and morphology. ${ }^{5}$ In addition, multiple lipomas are observed in the context of hereditary neoplastic syndromes including those associated with mutations in the MEN1 and PTEN genes (OMIM 131100 and 153480, respectively). Intriguingly, Li et al. ${ }^{4}$ observed that in patients with bilateral retinoblastoma the presence of lipomas may 
indicate a higher risk of second cancers. Therefore, the factors responsible for lipoma susceptibility in retinoblastoma may give a clue to the determinants of second cancer development in these patients. Here we present a large pedigree in which lipoma predisposition is linked to a mutant RB1 allele that causes low-penetrance retinoblastoma.

\section{Materials and methods Patients}

The family was ascertained through individual IV-8, who is the only one affected with bilateral retinoblastoma (Figure 1). A total of four other relatives developed unilateral retinoblastoma. Average age at diagnosis was 10 months (range 3 to 24 months). Individuals affected with retinoblastoma were connected through seven unaffected obligate carriers. Ophthalmologic investigation in individuals III-1, III-5, III-14, III-16, III-17, IV-1, IV-2, IV-5, IV-6, and IV-7 showed no evidence of retinoma. With two exceptions (III-5 and III-12) carriers without retinoblastoma have multiple $(\geqslant 2)$ subcutaneous lipomas, preferentially located on the face, neck, shoulders and upper chest. Multiple lipomas were also present in three of the retinoblastoma patients as well as in several other family members past the age of 35 years. In all individuals who are alive, the presence of lipomas was either affirmed following surgical excision or verified by clinical examination. Additional extraocular tumours were observed in four family members: nuchal fibromas in individuals II-1 and III-1, lung cancer in II-2, and Hodgkin lymphoma in III-6.
Information on the phenotypic status of deceased family members is based on medical history.

\section{Methods}

Blood samples were collected from the family members with appropriate consent and DNA extracted according to standard protocols. For linkage analysis, polymorphic short tandem repeat loci on chromosome 13 were investigated by PCR with fluorescent-labelled primers and Genescan analysis (ABI 310 Genetic Analyzer, ABI, Foster City, USA). The names and genetic locations of these loci are shown in Figure 1. Two-point linkage analysis was performed using the LINKAGE program. Cytogenetic analysis of peripheral blood lymphocytes was performed using standard methods. For mutation analysis in the RB1 gene, all coding parts of exons and flanking splice sites were amplified from peripheral blood DNA by PCR and examined by sequencing as described. ${ }^{6}$ For analysis of transcripts of the RB1 gene, total RNA was purified from lymphoblastoid cell lines using the RNAeasy protocol (Qiagen, Hilden, Germany) and reverse transcribed primed by random oligos (GeneAmp RNA PCR Kit, Perkin Elmer). For PCR amplification and sequencing of cDNA, primers RBc9se, RBc11se, RBc13se, and RBc16as were used as described. ${ }^{7}$ A mismatched-primer PCR-RFLP strategy was established ${ }^{8}$ using specifically designed primers RB13mmse (5'-GGGTTGTGTCGAAATTGGATCTC-3') and RB13mmas (5'-CACAGGCAGCAGGGATATAG-3'). In two separate assays, restriction

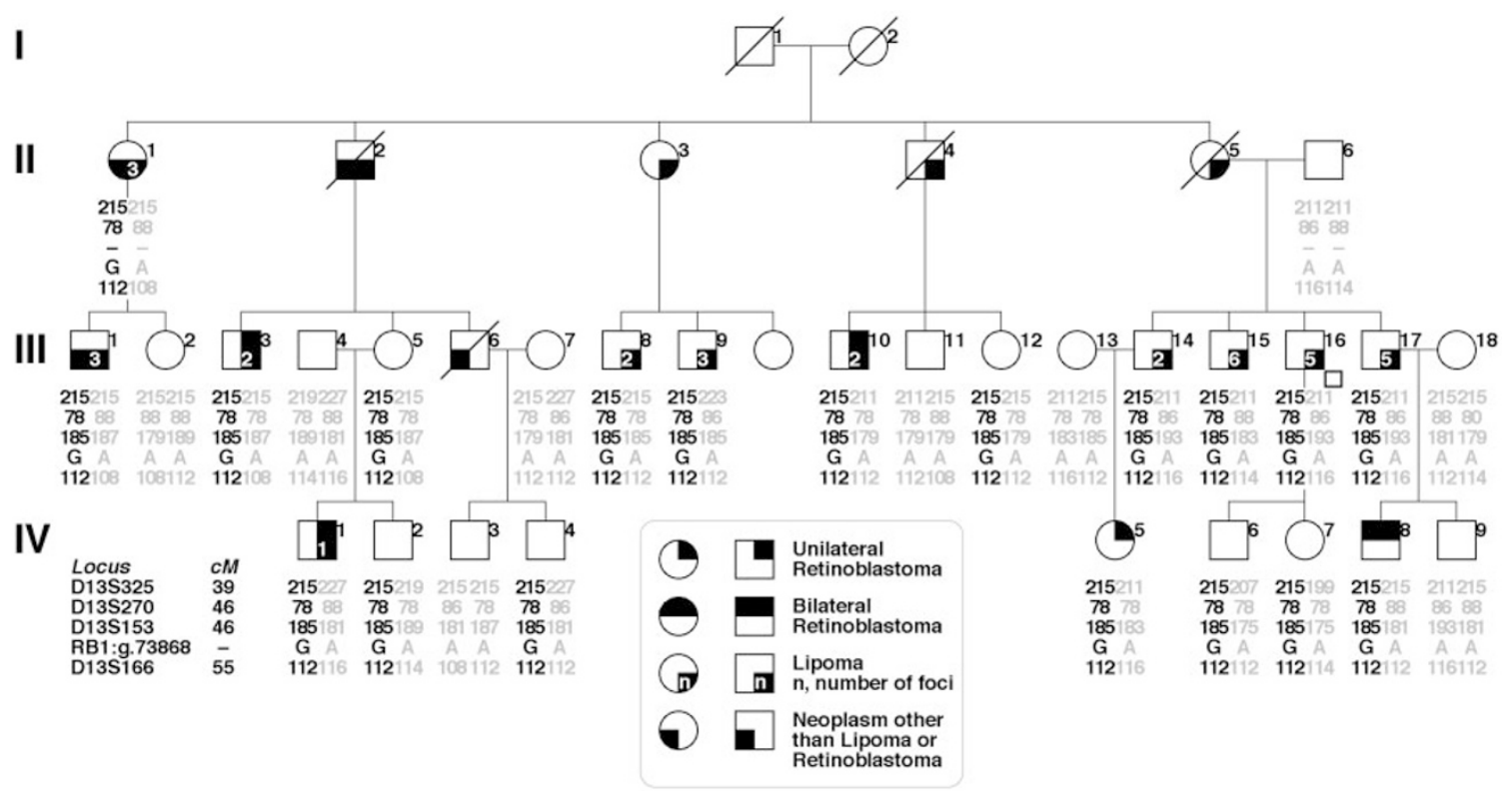

Figure 1 Pedigree of the family cosegregating predisposition to retinoblastoma and multiple lipomatosis. The results of genotyping of polymorphic loci linked to the RB1 gene and the mutational status are given below the symbols representing the individual family members. Map position of loci according to Broman et al. ${ }^{13}$ 
endonucleases $A v a \mathrm{I}$ and DdeI were used to identify the presence of the g.73868G (mutant) and g.73868A (normal) allele, respectively.

\section{Results}

In order to determine if retinoblastoma predisposition and multiple lipomatosis co-segregate with the RB1 gene on chromosome 13q14, we investigated linked polymorphic loci in peripheral blood DNA from 28 family members, including five unrelated spouses. A shared haplotype spanning approximately $16 \mathrm{cM}$ was present in all retinoblastoma patients, obligate carriers, and other tested individuals with multiple lipomas (Figure 1). Cytogenetic analysis of lympho- cytes from peripheral blood of the proband (IV-8) had shown a normal male karyotype. Therefore, we searched for a predisposing RB1 gene mutation by sequencing of all coding parts of exons and flanking splice sites. We found a heterozygous $A>G$ substitution at the penultimate position of exon 13 (g.73868A $>$ G). This alteration was not identified in more than 100 normal chromosomes. Sequence analysis of peripheral blood DNA from IV-1, IV-2, and IV-5 also showed this mutation. Apparently, the transition produces a missense change in codon 444 (Gln to Arg). However, the function of the $5^{\prime}$-splice site of intron 13 is sensitive to mutations in the preceding exon. ${ }^{7}$ Therefore, we investigated RNA isolated from lymphoblastoid cell lines derived from individuals III-17, IV-1, IV-2, IV-8, and two controls. Agarose

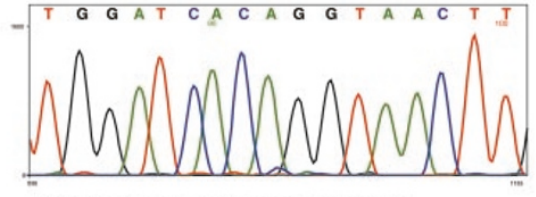

peripheral blood DNA, wild type

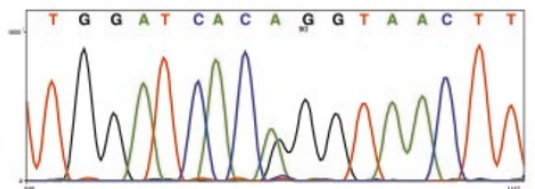

peripheral blood DNA,patient IV-8

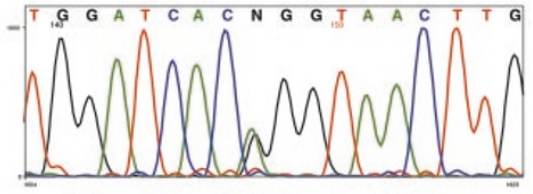

A peripheral blood DNA,patient IV-1

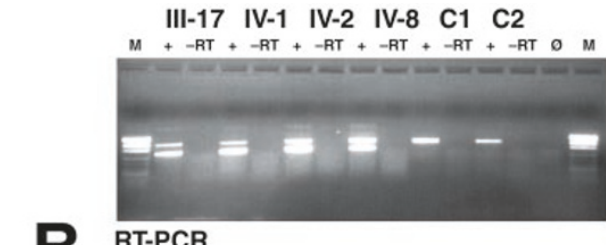

18

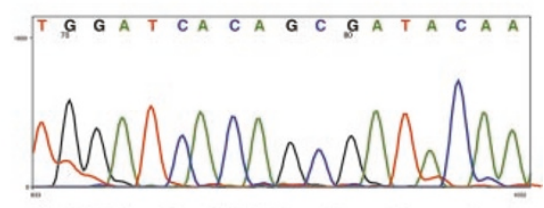

RT-PCR patient III-17, wt-length product

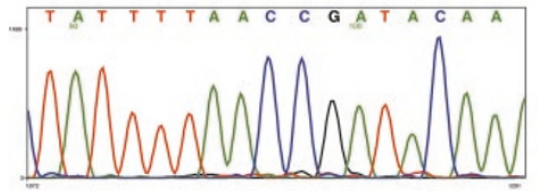

RT-PCR patient III-17, short product

\section{IATCACAGCGATACAA/}

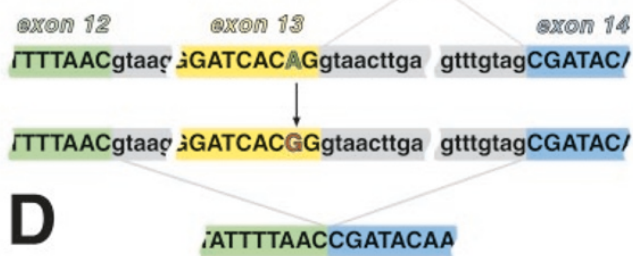

Figure 2 (A) Result of sequence analysis of exon 13 PCR products from genomic DNA from peripheral blood of a normal control, patient IV-8, and patient IV-1 of the family. Patients are heterozygous for the wild type A and mutant $\mathrm{G}$ at the penultimate nucleotide of exon 13 . (B) RT - PCR on RNA isolated from lymphoblastoid cell lines derived from individuals III-17, IV-1, IV-2, IV-8 (all heterozygous for g.73868A $>\mathrm{G}$ ), and two controls ( $\mathrm{C} 1$ and $\mathrm{C} 2$ ) with primers spanning exons 12 to 15 of the RB1 gene. All carriers show products of normal size and a smaller band. Controls only show a products of wild-type length. The DNA size marker (M) is a Mspl digest of pUC19 DNA with fragments of 501/489, 404, 331, 242, 190, 147, 111/110, and $67 \mathrm{bp}$. (C) Result of sequence analysis of individual excised RT-PCR products from patient III-17. The normal sized RT-PCR products show the regular spliced transcript sequence, while exon 13 is skipped in the small RT-PCR product. (D) Schematic representation showing the mutation identified in the family presented here and the regular and mutant transcript sequences. 
gel electrophoresis of RT-PCR products from samples with the $\mathrm{g} .73868 \mathrm{~A}>\mathrm{G}$ mutation showed normal-sized and smaller products (Figure 2B). Sequence analysis of the individual DNA bands showed that the smaller products lack $117 \mathrm{bp}$ corresponding to exon 13 (in-frame loss of codons 406 to 444), while the products of expected length showed the normal RB1 cDNA sequence. Specifically, the penultimate nucleotide of exon 13 in the regularly spliced product only shows the wild-type base. Therefore, correctly spliced transcripts of the mutant allele-if present at all-are below the detection limits of the BigDye terminator sequencing chemistry used here. ${ }^{9}$ Using a mismatched-primer PCR-RFLP strategy, heterozygosity for the mutant allele (g.73868G) was confirmed in all living family members who shared the haplotype cosegregating with the retinoblastoma/multiple lipomatosis phenotype. Archival material from the nuchal fibroma of patient III-1 showed no loss of the normal allele (data not shown). A total of 24 heterozygous carriers of the g.73868A allele were ascertained through pedigree evaluation or mutation analysis. Only five of them had developed retinoblastoma during childhood (observed penetrance $\mathrm{RB}_{\mathrm{RB}}$ $\approx 0.21$ ). By contrast, multiple lipomas were present in 13 out of those 15 at-risk subjects, who were older than 35 years and for whom results of DNA analysis and/or reliable clinical information were available (observed penetrance ${ }_{\mathrm{ML}} \approx 0.87$ ). Co-segregation between the multiple lipomatosis phenotype and the mutant RB1 allele was formally demonstrated by two-point linkage analysis on generations I to III, using the LINKAGE program. The maximum LOD score value was 3.12 at $\theta=0$, assuming a penetrance of 0.87 . We also noticed a statistically significant segregation distortion in favour of the mutant allele $(P<0.005)$.

\section{Discussion}

In the pedigree presented here autosomal dominant transmission of multiple lipomas is linked to a mutant RB1 allele. Age at presentation and site distribution of lipomas compare with the phenotype reported in patients with hereditary retinoblastoma. ${ }^{10,11}$ However, in this family almost all carriers of the mutant RB1 allele developed multiple lipomas while lipomas are infrequent in other patients with hereditary predisposition to retinoblastoma. It is important to note that the presentation of lipomas on the background of retinoblastoma predisposition is distinct from that observed in the two known lipomatoses, ie familial multiple lipomatosis or benign cervical lipomatosis. Specifically, individual lipomas in the family reported here were discrete and movable as in familial multiple lipomatosis but very unlike the diffuse fatty infiltration typically seen in benign cervical lipomatosis. ${ }^{5}$ On the other hand, lipomas in hereditary retinoblastoma are preferentially located on the face, neck, shoulders and upper chest. These locations are commonly affected in benign cervical lipomatosis but are mostly spared in familial multiple lipomatosis. Moreover, molecular analysis of a two generation pedigree with typical familial multiple lipomatosis provided evidence against linkage to polymorphic loci on chromosome 13q14 (data not shown). Nevertheless we cannot exclude that there is a distinct lipoma locus linked to the RB1 gene and that the lipoma-retinoblastoma family presented here is simply due to fortuitous co-segregation of mutations at these two loci that independently confer predisposition to lipoma and retinoblastoma. However, this explanation cannot account for the reported excess of lipomas among patients with hereditary retinoblastoma.

Intriguingly, the g.78868G mutation in the RB1 gene that was identified in the lipoma-retinoblastoma family has been reported in another family with retinoblastoma. ${ }^{12}$ In the reported pedigree, penetrance for retinoblastoma was as low as in the family presented here but none of the eight carriers of the mutation past 35 years showed evidence of lipomas $(\mathrm{H}$ Scheffer, personal communication). The phenotypic diversity associated with this mutation is not in keeping with the hypothesis that lipoma predisposition is caused by specific oncogenic RB1 mutations. ${ }^{4}$ Apparently, this mutation by itself is not sufficient to account for lipoma predisposition. In an alternative model we propose that a linked polymorphism-intra or extragenic-interferes with the expression of oncogenic RB1 gene mutations thus acting as a modifying factor. According to this hypothesis, specific alleles of the modifier can cause lipoma predisposition in carriers of an oncogenic RB1 gene mutation. This model can account both for cosegregation of multiple lipomatosis and retinoblastoma predisposition in the family presented here and for the excess of lipomas observed in patients with hereditary retinoblastoma.

\section{Acknowledgments}

We thank Hans Scheffer, Ruth Kleinerman for providing us with details on previously reported data and Giovanni Neri, Bernhard Horsthemke, Ruth Kleinerman, and Thomas Wienker for discussion.

\section{References}

1 Lohmann DR: RB1 gene mutations in retinoblastoma. Hum Mut 1999; 14: $283-288$.

2 Eng C, Li FP, Abramson DH et al: Mortality from second tumors among long-term survivors of retinoblastoma. J Natl Cancer Inst 1993; 85: $1121-1128$.

3 Blanquet V, Turleau C, Gross-Morand S, Sénamaud-Beaufort C, Doz F, Besmond C: Spectrum of germline mutations in the RB1 gene: a study of 232 patients with hereditary and non hereditary retinoblastoma. Hum Molec Genet 1995; 4: 383-388.

4 Li FP, Abramson DH, Tarone RE, Kleinerman RA, Fraumeni Jr JF, Boice Jr JD: Hereditary retinoblastoma, lipoma, and second primary cancers. J Natl Cancer Inst 1997; 89: 83 - 84 .

5 Leffell DJ, Braverman IM: Familial multiple lipomatosis. Report of a case and a review of the literature. J Am Acad Dermatol 1986; 15: $275-279$.

6 Lohmann DR, Brandt B, Höpping W, Passarge E, Horsthemke B: Spectrum of RB1 germ-line mutations in hereditary retinoblastoma. Am J Hum Genet 1996; 58: 940-949. 
7 Lohmann DR, Gerick M, Brandt B et al: Constitutional RB1-gene mutations in patients with isolated unilateral retinoblastoma. Am J Hum Genet 1997; 61: 282-294.

8 Haliassos A, Chomel JC, Grandjouan S, Kruh J, Kaplan JC, Kitzis A: Detection of minority point mutations by modified PCR technique: a new approach for a sensitive diagnosis of tumorprogression markers. Nucleic Acids Res 1989; 17: 8093 -8099.

9 Rosenblum BB, Lee LG, Spurgeon SL et al: New dye-labeled terminators for improved DNA sequencing patterns. Nucleic Acids Res 1997; 25: 4500-4504.

10 Li Y, Graham C, Lacy S, Duncan AM, Whyte P: The adenovirus E1A-associated $130-\mathrm{kD}$ protein is encoded by a member of the retinoblastoma gene family and physically interacts with cyclins A and E. Genes Dev 1993; 7: 2366-2377.
11 Rieder H, Lohmann D, Poensgen B et al: Loss of heterozygosity of the retinoblastoma (RB1) gene in lipomas from a retinoblastoma patient. J Natl Cancer Inst 1998; 90: 324-326.

12 Scheffer H, Van Der Vlies P, Burton M et al: Two novel germline mutations of the retinoblastoma gene (RB1) that show incomplete penetrance, one splice site and one missense. $J$ Med Genet 2000; 37: E6.

13 Broman KW, Murray JC, Sheffield VC, White RL, Weber JL: Comprehensive human genetic maps: individual and sexspecific variation in recombination. Am J Hum Genet 1998; 63: $861-869$. 